Title : will be set by the publisher

Editors : will be set by the publisher

EAS Publications Series, Vol. ?, 2018

\title{
BLAZHKO EFFECT IN DOUBLE MODE CEPHEIDS
}

\author{
P. Moskalik ${ }^{1}$ and Z. Kołaczkowski ${ }^{2}$
}

\begin{abstract}
Systematic survey for multiperiodicity in the LMC Cepheids (Moskalik, Kołaczkowski \& Mizerski 2004, 2006) has led to discovery of several new forms of pulsational behaviour. One of them is periodic amplitude and phase modulation observed in many first/second overtone (FO/SO) double mode Cepheids. In the current paper we present detailed discussion of this newly discovered phenomenon, based on a combined OGLE+MACHO sample of double mode pulsators.
\end{abstract}

\section{Time Series Analysis}

We searched for additional signal in the data using a standard consecutive prewhitening technique. To that effect, we first fitted the data with the double frequency Fourier sum representing pulsations in two radial modes:

$$
m(t)=\langle m\rangle+\sum_{j, k} \mathrm{~A}_{j k} \sin \left[2 \pi\left(j \mathrm{f}_{1}+k \mathrm{f}_{2}\right) t+\phi_{j k}\right] .
$$

The frequencies of the modes, $\mathrm{f}_{1}$ and $\mathrm{f}_{2}$, were also optimized. The residuals of the fit were then searched for additional periodicities. This was done with the Fourier transform, calculated over the range of $0-5 \mathrm{c} / \mathrm{d}$. In the next step, a new Fourier fit with all frequencies discovered so far was performed and the fit residuals were searched for additional frequencies again. The process was stopped when no new frequencies were detected.

\section{LMC Sample}

Our initial analysis was performed with DIA-reduced OGLE-II photometry (Żebruń et al. 2001). There are $56 \mathrm{FO} / \mathrm{SO}$ double mode Cepheids in the OGLE catalog (Soszyński et al. 2000). In one-third of these stars we detected residual

\footnotetext{
${ }^{1}$ Copernicus Astronomical Center, Bartycka 18, 00-716 Warsaw, Poland

${ }^{2}$ Universidad de Concepcion, Departamento de Fisica, Casilla 160-C, Concepcion, Chile
}

(c) EDP Sciences 2018

DOI: (will be inserted later) 
Title : will be set by the publisher

Table 1. Blazhko FO/SO Double-Mode Cepheids in the LMC

\begin{tabular}{|c|c|c|c|c|c|c|c|c|c|}
\hline $\begin{array}{l}\text { OGLE ID/ } \\
\text { /MACHO ID }\end{array}$ & $\begin{array}{c}\mathrm{P}_{1} \\
{[\text { day }]}\end{array}$ & $\begin{array}{c}\mathrm{P}_{2} \\
{[\text { day }]}\end{array}$ & $\begin{array}{c}\mathrm{P}_{\mathrm{B}} \\
{[\text { day }]}\end{array}$ & $\begin{array}{c}A_{1} \\
{[\mathrm{mag}]}\end{array}$ & $\begin{array}{c}A_{1}^{-} \\
A_{1}^{+} \\
{[\mathrm{mag}]}\end{array}$ & $\begin{array}{l}A_{1}^{--} \\
A_{1}^{++} \\
{[\mathrm{mag}]}\end{array}$ & $\begin{array}{c}A_{2} \\
{[\mathrm{mag}]}\end{array}$ & $\begin{array}{c}A_{2}^{-} \\
A_{2}^{+} \\
{[\mathrm{mag}]}\end{array}$ & $\begin{array}{c}A_{2}^{--} \\
A_{2}^{++} \\
{[\mathrm{mag}]}\end{array}$ \\
\hline SC1-44845 & 0.9520 & 0.7660 & 794.0 & 0.165 & $\begin{array}{l}0.021 \\
0.011\end{array}$ & - & 0.046 & $\begin{array}{l}0.021 \\
0.010\end{array}$ & - \\
\hline SC1-285275 & 0.8566 & 0.6892 & 891.6 & 0.177 & $\begin{array}{l}0.031 \\
0.014\end{array}$ & - & 0.040 & $\begin{array}{l}0.022 \\
0.013\end{array}$ & $\begin{array}{l}0.008 \\
-\end{array}$ \\
\hline SC1-335559 & 0.7498 & 0.6036 & 779.2 & 0.227 & $\begin{array}{c}0.019 \\
-\end{array}$ & - & 0.062 & $\begin{array}{c}0.023 \\
-\end{array}$ & - \\
\hline SC2-55596 & 0.9325 & 0.7514 & 768.2 & 0.146 & $\begin{array}{l}0.007 \\
0.007\end{array}$ & - & 0.039 & - & - \\
\hline SC6-142093 & 0.8963 & 0.7221 & 1101.6 & 0.154 & $\begin{array}{l}0.028 \\
0.013\end{array}$ & - & 0.043 & $\begin{array}{l}0.017 \\
0.017\end{array}$ & $\begin{array}{l}0.012 \\
0.006\end{array}$ \\
\hline SC6-267410 & 0.8885 & 0.7168 & 856.9 & 0.120 & - & - & 0.036 & $\overline{0.033}$ & - \\
\hline SC8-10158 & 0.6900 & 0.5557 & 1060.7 & 0.175 & $\begin{array}{c}0.011 \\
-\end{array}$ & - & 0.031 & $\begin{array}{l}0.019 \\
0.012\end{array}$ & - \\
\hline SC11-233290 & 1.2175 & 0.9784 & 1006.2 & 0.186 & $\begin{array}{l}0.019 \\
0.010\end{array}$ & - & 0.043 & $\begin{array}{l}0.017 \\
0.010\end{array}$ & $\begin{array}{c}0.006 \\
-\end{array}$ \\
\hline SC15-16385 & 0.9904 & 0.7957 & 1123.1 & 0.258 & $\begin{array}{l}0.017 \\
0.016\end{array}$ & - & 0.049 & $\begin{array}{c}0.020 \\
-\end{array}$ & - \\
\hline SC20-112788 & 0.7377 & 0.5943 & 1379.2 & 0.164 & $\begin{array}{l}0.062 \\
0.019\end{array}$ & $\begin{array}{c}0.022 \\
-\end{array}$ & 0.015 & $\begin{array}{l}0.016 \\
0.013\end{array}$ & - \\
\hline SC20-138333 & 0.8598 & 0.6922 & 795.0 & 0.189 & $\begin{array}{l}0.013 \\
0.008\end{array}$ & - & 0.060 & $\begin{array}{l}0.018 \\
0.010\end{array}$ & - \\
\hline 2.4909 .67 & 1.0841 & 0.8700 & 1019.7 & 0.216 & $\begin{array}{l}0.012 \\
0.010\end{array}$ & - & 0.055 & $\begin{array}{l}0.013 \\
0.009\end{array}$ & - \\
\hline 13.5835 .55 & 0.8987 & 0.7228 & 1074.9 & 0.244 & $\begin{array}{l}0.037 \\
0.021\end{array}$ & $\begin{array}{c}0.010 \\
-\end{array}$ & 0.040 & $\begin{array}{l}0.026 \\
0.016\end{array}$ & $\begin{array}{c}0.012 \\
-\end{array}$ \\
\hline 14.9585 .48 & 0.9358 & 0.7528 & 1092.5 & 0.139 & $\begin{array}{l}0.024 \\
0.008\end{array}$ & - & 0.036 & $\begin{array}{l}0.025 \\
0.010\end{array}$ & $\begin{array}{c}0.012 \\
-\end{array}$ \\
\hline 17.2463 .49 & 0.7629 & 0.6140 & 1069.9 & 0.235 & $\begin{array}{l}0.018 \\
0.014\end{array}$ & - & 0.051 & $\begin{array}{l}0.013 \\
0.015\end{array}$ & - \\
\hline 18.2239 .43 & 1.3642 & 1.0933 & 706.8 & 0.230 & $\begin{array}{l}0.035 \\
0.014\end{array}$ & $\begin{array}{c}0.010 \\
-\end{array}$ & 0.063 & $\begin{array}{l}0.017 \\
0.015\end{array}$ & - \\
\hline 22.5230 .61 & 0.6331 & 0.5101 & 804.3 & 0.221 & - & - & 0.045 & $\begin{array}{l}0.016 \\
0.014\end{array}$ & - \\
\hline 23.2934 .45 & 0.7344 & 0.5918 & 797.6 & 0.207 & $\begin{array}{c}0.023 \\
-\end{array}$ & - & 0.055 & $\begin{array}{l}0.031 \\
0.026\end{array}$ & $\begin{array}{l}0.015 \\
0.011\end{array}$ \\
\hline 23.3184 .74 & 0.8412 & 0.6778 & 1126.0 & 0.160 & $\begin{array}{c}0.015 \\
-\end{array}$ & - & 0.048 & $\begin{array}{l}0.011 \\
0.013\end{array}$ & - \\
\hline 80.7080 .2618 & 0.7159 & 0.5780 & 920.3 & 0.168 & $\begin{array}{c}0.010 \\
-\end{array}$ & - & 0.055 & $\begin{array}{l}0.006 \\
0.009\end{array}$ & - \\
\hline
\end{tabular}




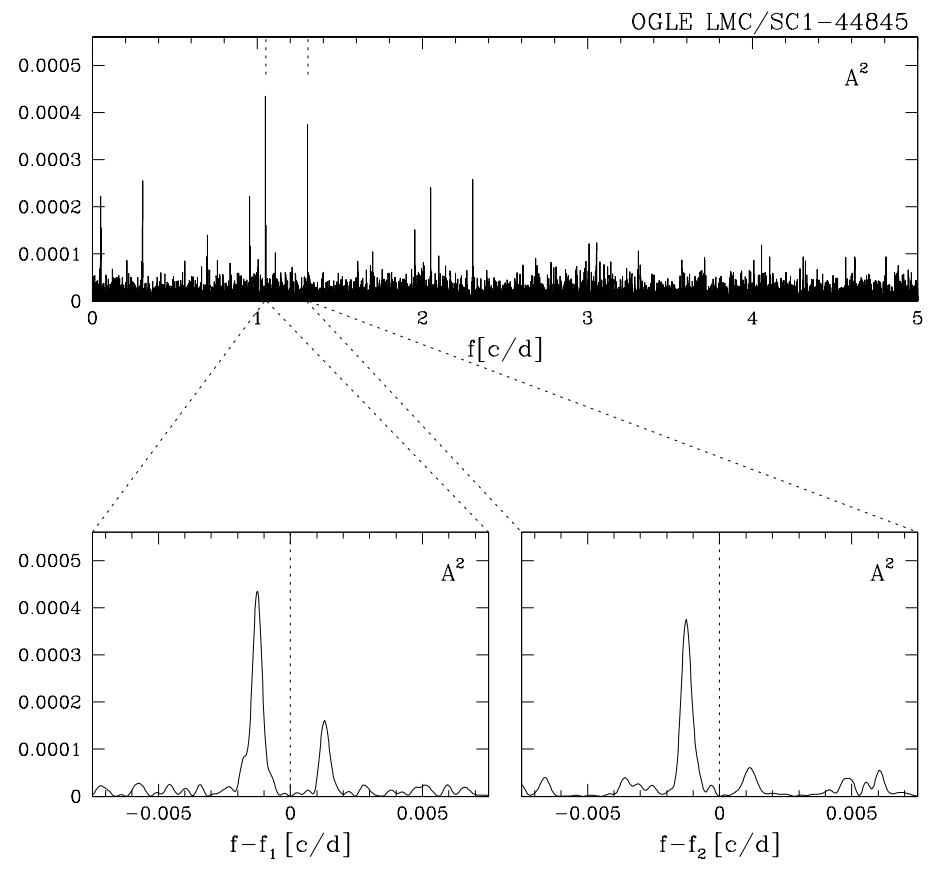

Fig. 1. Power spectrum of FO/SO double mode Cepheid LMC/SC1-44845 after prewhitening with frequencies of the two radial modes (and their linear combinations). Removed radial frequencies are indicated by dashed lines. Lower panels display the fine structure around the radial modes.

signal close to one or both primary (radial) pulsation frequencies. In all cases, however, the secondary frequencies were not resolved from the primary ones. Clearly, $\sim 1200$ days timebase provided by OGLE-II data was insufficient for our purpose. Therefore, the final frequency analysis was performed with MACHO photometry (Allsman \& Axelrod 2001), which had lower precision, but offered much longer timebase $(2700-2800$ days $)$.

The OGLE-II sample was supplemented by 51 additional FO/SO double mode Cepheids discovered by MACHO team outside the area covered by OGLE-II (Alcock et al. 1999, 2003). Our final FO/SO Cepheid sample consisted of 107 objects.

\section{Results}

Residual power close to the primary pulsation frequencies was detected in 37 FO/SO double mode Cepheids (35\% of the sample). In 20 of these stars, which constitute $19 \%$ of the entire sample, we were able to resolve this power into individual frequencies. We consider two frequencies to be resolved if $1 / \Delta \mathrm{f}<1400$ days. All resolved stars are listed in Table 1 . 
Title : will be set by the publisher

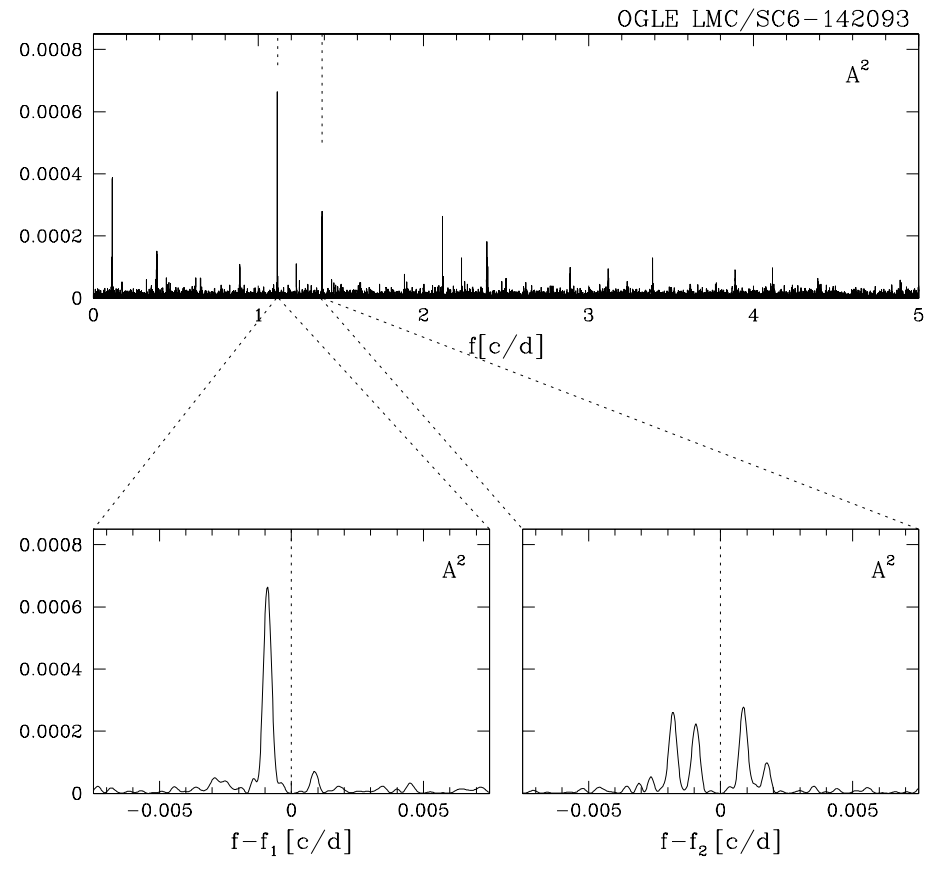

Fig. 2. Same as Fig.1 but for LMC/SC6-142093.

\subsection{Frequency Domain}

Resolved FO/SO double mode Cepheids display very characteristic frequency pattern. In most cases, we detect two peaks in the vicinity of each radial frequency. They are located on the opposite sides of the primary (radial) peak and together with the primary frequency they form an equally spaced frequency triplet. In majority of cases, such a structure is detected around both radial modes. Typical example of this pattern is displayed in Fig.1 In two Cepheids we detect equally spaced frequency quintuplets centered on the second overtone. One of these objects is presented in Fig.2 Incomplete quintuplets are also seen in 6 other stars.

The secondary peaks are always very small, with amplitudes of only $15 \mathrm{mmag}$ on average and never higher than $62 \mathrm{mmag}$. The separation of components in triplets/quintuplets, $\Delta \mathrm{f}$, is also very small and never exceed $0.00142 \mathrm{c} / \mathrm{d}$. This corresponds to the beat period of $\mathrm{P}_{\mathrm{B}}>700$ day. The lower limit for $\Delta \mathrm{f}$ (upper limit for $\mathrm{P}_{\mathrm{B}}$ ) is currently given by resolution of the data. The physical upper limit for the beat period $\mathrm{P}_{\mathrm{B}}$ is unknown.

When present around both radial modes, the two triplets/quintuplets have nearly the same frequency spacings. Specifically, the difference between the two spacings, $\left|\Delta \mathrm{f}_{2}-\Delta \mathrm{f}_{1}\right|$ is always below $9 \times 10^{-5} \mathrm{c} / \mathrm{d}$. According to simulations of Alcock et al. (2000, their Fig. 14), for MACHO data such a frequency difference is sta- 

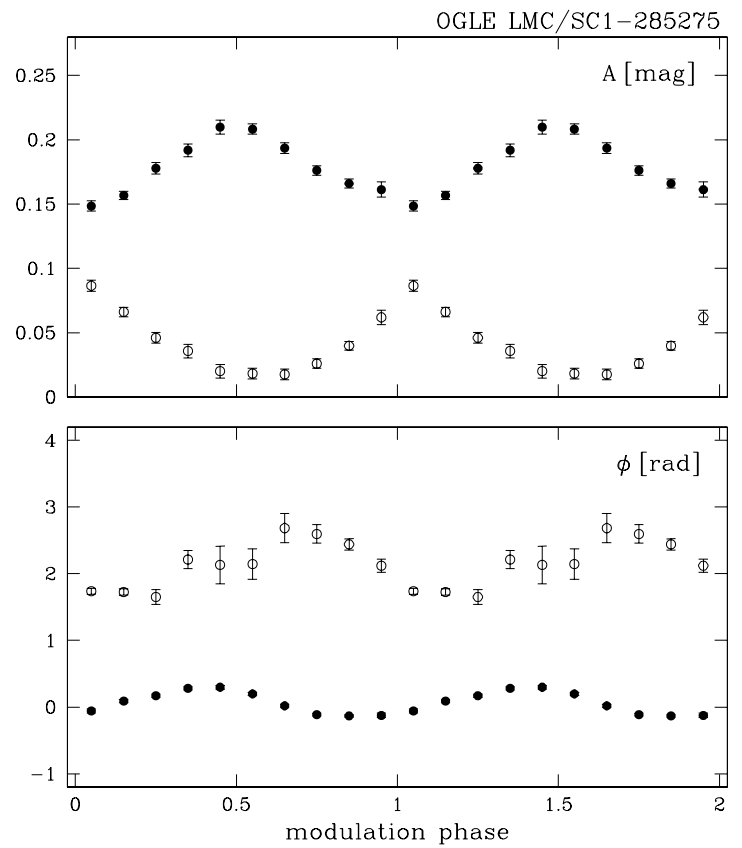

Fig. 3. Periodic modulation of FO/SO double mode Cepheid LMC/SC1-285275. First and second overtones are displayed with filled and open circles, respectively. Modulation period is $\mathrm{P}_{\mathrm{B}}=891.6$ day.

tistically consistent with no difference at all. In other words, triplets/quintuplets around both radial modes have the same frequency separation within accuracy of the data.

\subsection{Time Domain}

The triplet/quintuplet frequency pattern can be interpreted as a result of periodic modulation of amplitudes and/or phases of both radial modes, with common pe$\operatorname{riod} \mathrm{P}_{\mathrm{B}}=1 / \Delta \mathrm{f}$. In Fig. 3 we show this modulation for one of the stars. The plot has been constructed by dividing the data into 10 subsets, each spanning $10 \%$ of the modulation cycle, and then fitting radial modes and their linear combinations (Eq.(1)) to each subset separately. The resulting amplitudes and phases of the modes are plotted vs. modulation phase.

Fig. 3 shows that both amplitudes and phases undergo modulation. The amplitude variability is much stronger for the second than for the first overtone $(80 \%$ vs. $30 \%$ change). Minimum amplitude of one mode coincides with maximum amplitude of the other mode. Maximum phase of the first overtone occurs just before maximum of its amplitude. For the second overtone, maximum phase coincides with minimum amplitude. 
Title : will be set by the publisher
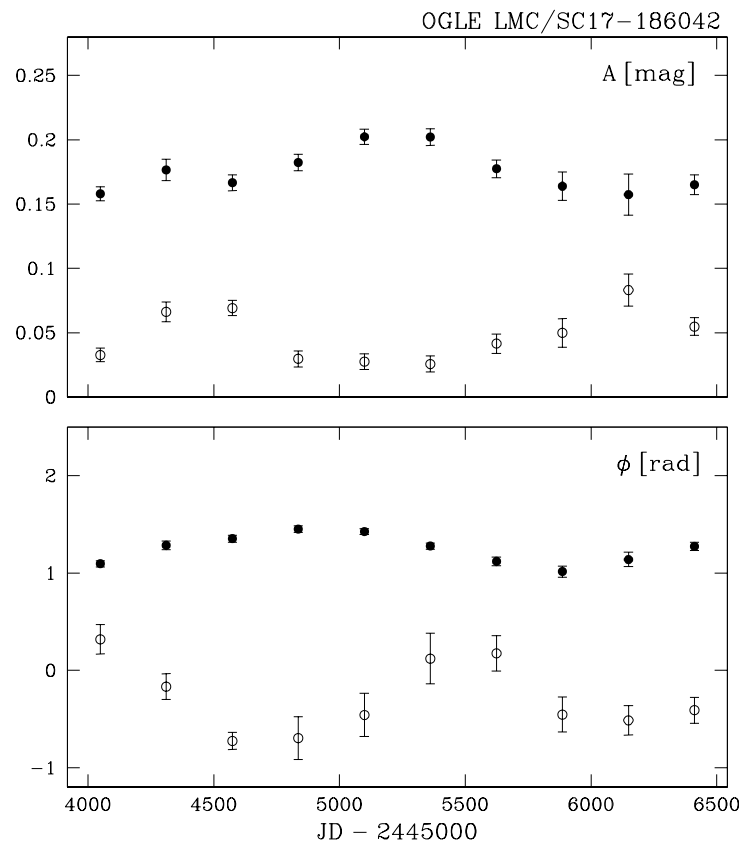

Fig. 4. Slow amplitude and phase variability of $\mathrm{FO} / \mathrm{SO}$ double mode Cepheid LMC/SC17-186042. First and second overtones are displayed with filled and open circles, respectively.

Characteristic pattern of periodic modulation displayed in Fig. 3 is common to all Cepheids listed in Table1. The phenomenon is similar to the Blazhko effect in RR Lyrae stars (e.g. Kurtz et al. 2000). By analogy, variables of Table1 can be called "Blazhko double mode Cepheids".

\subsection{Stars with Unresolved Power Spectrum}

In $17 \mathrm{FO} / \mathrm{SO}$ double mode Cepheids ( $16 \%$ of the sample) we find after prewhitening a significant residual power unresolved from the primary pulsation frequencies. This is a signature of slow amplitude and/or phase variability, not resolved within the length of available data (i.e. 2700 days).

For closer examination of this slow variability we have performed for each star a similar analysis to that presented in Fig. 3. Specifically, we have divided the data into 10 subsets, each spanning $10 \%$ of the total timebase, and then have fitted Eq.(1) separately to each subset. In some stars we have detected only changes of pulsation phases, but in 10 variables we have also detected clear changes of the amplitudes. In Fig.4 we show typical example of such behaviour. The pattern of amplitude and phase variability is very much the same as that displayed in Fig. 3. In particular, the amplitudes of the two radial modes vary in opposite directions. 
Table 2. Unresolved FO/SO Double-Mode Cepheids in the LMC

\begin{tabular}{lcclcc}
\hline MACHO Star & $P_{1}$ [day] & $P_{2}$ [day] & OGLE Star & $P_{1}$ [day] & $P_{2}$ [day] \\
\hline 6.6934 .67 & 0.9202 & 0.7400 & SC4-176400 & 1.1089 & 0.8951 \\
11.9348 .78 & 0.7378 & 0.5944 & SC4-220148 & 0.7408 & 0.5951 \\
14.9098 .35 & 0.7278 & 0.5865 & SC7-120511 & 1.2511 & 1.0037 \\
15.10428 .60 & 0.6518 & 0.5253 & SC10-204083 & 0.5263 & 0.4229 \\
23.3061 .82 & 0.5957 & 0.4810 & SC16-266808 & 1.3529 & 1.0810 \\
24.2853 .69 & 0.7088 & 0.5717 & SC17-186042 & 0.6110 & 0.4912 \\
24.2855 .80 & 0.5938 & 0.4783 & SC20-188572 & 1.0152 & 0.8145 \\
47.2127 .102 & 0.5785 & 0.4666 & SC $21-12012$ & 1.3415 & 1.0749 \\
80.7079 .62 & 1.3479 & 1.0764 & & & \\
\hline
\end{tabular}

This similarity suggests, that at least some of the unresolved FO/SO double mode Cepheids experience the same type of periodic modulation as Cepheids listed in Table[1, but on timescales longer than the current data.

\section{What Causes the Modulation ?}

Periodic amplitude and phase modulation in FO/SO double mode Cepheids is by no mean a rare phenomenon. According to our analysis its incidence rate is at least $19 \%$ and perhaps as high as $35 \%$.

Any model of modulated double mode Cepheids has to explain two most basic properties of these stars:

- both radial modes are modulated with the same period.

- amplitudes of the two modes change in opposite phase: maximum of one amplitude coincides with minimum of the other.

Two models have been proposed to explain Blazhko modulation in RR Lyrae stars: the oblique magnetic pulsator model (Shibahashi 1995, 2000) and 1:1 resonance model (Nowakowski \& Dziembowski 2001). We will argue, that both these models fail to account for modulation observed in the FO/SO double mode Cepheids.

The oblique magnetic pulsator model assumes presence of a dipole magnetic field, which is inclined to the rotation axis of the star. The field introduces distortion to the radial pulsation. As the star rotates, this distortion is viewed from different angles, causing variation of the pulsation amplitude. However, in this scenario both modes should display maximum amplitude simultaneously, because they are both distorted in the same way. This is not what we observe.

The model proposed by Nowakowski \& Dziembowski (2001) assumes resonant coupling of a radial mode to nonradial modes of $\ell=1$. Such a mechanism generates in a natural way a triplet of equally spaced frequencies. The beating of these 
Title : will be set by the publisher

frequencies causes apparent amplitude and phase modulation. Generalizing this model to coupling with modes of $\ell=2$, we can also explain frequency quintuplets. However, in this resonance model, modulation of each radial mode is an independent effect. Therefore, there is no reason why both modes should be modulated with the same period. There is also no reason why amplitude variations of the modes should be in any particular phase relation to each other (as is observed). Finally, the 1:1 resonance model does not explain why in vast majority of cases modulation is observed either in both radial modes or in none of them.

At this stage, the mechanism causing amplitude and phase modulation of FO/SO double mode Cepheids remains unknown. Nevertheless, the available observations already provide some important clues. The fact that the two modes are always modulated with the same period proves that their behaviour is not independent. Both modes must be part of the same dynamical system. The fact that high amplitude of one mode always coincides with low amplitude of the other mode strongly suggests that energy transfer between the two modes is involved. Thus, available evidence clearly points towards some form of mode coupling in which both radial modes take part.

Acknowledgements. This work has been supported by the Polish MNiSW Grant No. 1 P03D 01130.

\section{References}

Alcock, C., Allsman, R. A., Alves, D. R., et al. 1999, ApJ, 511, 185

Alcock, C., Allsman, R. A., Alves, D. R., et al. 2000, ApJ, 542, 257

Alcock, C., Alves, D. R., Becker, A. et al. 2003, ApJ, 598, 597

Allsman, R. A. \& Axelrod, T. S. 2001, astro-ph/0108444

Kurtz, D. W., Alcock, C., Allsman, R. A., et al. 2000, in L. Szabados \& D. W. Kurtz eds., The Impact of Large-Scale Surveys on Pulsating Star Research, IAU Coll. 176, ASP Conference Series, 203, 291

Moskalik, P., Kołaczkowski, Z. \& Mizerski, T. 2004, in D. W. Kurtz \& K. Pollard eds., Variable Stars in the Local Group, IAU Coll. 193, ASP Conference Series, 310, 498

Moskalik, P., Kołaczkowski, Z. \& Mizerski, T. 2006, in A. R. Walker \& G. Bono eds., Stellar Pulsation and Evolution, Mem. Soc. Astron. Ital., 77, 563.

Nowakowski, R. M. \& Dziembowski, W. A. 2001, Acta Astron., 51, 5

Shibahashi, H. 1995, in R. K. Ulrich, E. J. Rhodes Jr. \& W. Däppen eds., GONG'94: Helio- and Asteroseismology from Earth and Space, ASP Conference Series, 76, 618

Shibahashi, H. 2000, in L. Szabados \& D. W. Kurtz eds., The Impact of Large-Scale Surveys on Pulsating Star Research, IAU Coll. 176, ASP Conference Series, 203, 299

Soszyński, I., Udalski, A., Szymański, M., et al. 2000, Acta Astron., 50, 451

Żebruń, K., Soszyński, I., Woźniak, P. R. et al. 2001, Acta Astron., 51, 317 